\title{
CE-MS metabolic profiling of volume-restricted plasma samples from an acute mouse model for epileptic seizures to discover potentially involved metabolomic features
}

\author{
Karen Segers, ${ }^{\mathrm{a}, \mathrm{b}, \#}$, Wei Zhang ${ }^{\mathrm{c}, \#}$, Najat Aourz ${ }^{\mathrm{b}}$, Jana Bongaerts ${ }^{\mathrm{a}, \mathrm{b}}$, Sven Declerck $^{\mathrm{a}}$, \\ Debby Mangelings ${ }^{\mathrm{a}}$, Thomas Hankemeier ${ }^{\mathrm{c}}$, Dimitri De Bundel ${ }^{\mathrm{b}}$, Yvan Vander Heyden ${ }^{\mathrm{a}}$, \\ Ilse Smolders ${ }^{\mathrm{b}}$, Rawi Ramautar ${ }^{\mathrm{c}, 1}$, Ann Van Eeckhaut ${ }^{\mathrm{b}, *, 1}$ \\ a Department of Analytical Chemistry, Applied Chemometrics and Molecular Modelling, Vrije Universiteit Brussel, Laarbeeklaan 103, 1090 Brussels, Belgium \\ b Department of Pharmaceutical Chemistry, Drug Analysis and Drug Information, Center for Neurosciences (C4N), Vrije Universiteit Brussel, Laarbeeklaan 103, 1090, \\ Brussels, Belgium \\ ${ }^{\mathrm{c}}$ Biomedical Microscale Analytics, Division of Systems Biomedicine and Pharmacology, Leiden Academic Centre for Drug Research (LACDR), Leiden University, \\ Einsteinweg 55, 2333, CC Leiden, the Netherlands
}

\section{A R T I C L E I N F O}

\section{Keywords:}

Capillary electrophoresis

Epileptic seizures

Mass Spectrometry

Metabolomics

Volume-restricted plasma samples

\begin{abstract}
A B S T R A C T
Currently, a high variety of analytical techniques to perform metabolomics is available. One of these techniques is capillary electrophoresis coupled to mass spectrometry (CE-MS), which has emerged as a rather strong analytical technique for profiling polar and charged compounds. This work aims to discover with CE-MS potential metabolic consequences of evoked seizures in plasma by using a $6 \mathrm{~Hz}$ acute corneal seizure mouse model. CE-MS is an appealing technique because of its capability to handle very small sample volumes, such as the $10 \mu \mathrm{L}$ plasma samples obtained using capillary microsampling in this study. After liquid-liquid extraction, the samples were analyzed with CE-MS using low-pH separation conditions, followed by data analysis and biomarker identification. Both electrically induced seizures showed decreased values of methionine, lysine, glycine, phenylalanine, citrulline, 3-methyladenine and histidine in mice plasma. However, a second provoked seizure, 13 days later, showed a less pronounced decrease of the mean concentrations of these plasma metabolites, demonstrated by higher fold change ratios. Other obtained markers that can be related to seizure activities based on literature data, are isoleucine, serine, proline, tryptophan, alanine, arginine, valine and asparagine. Most amino acids showed relatively stable plasma concentrations between the basal levels (Time point 1) and after the 13-day wash-out period (Time point 3), which suggests its effectiveness. Overall, this work clearly demonstrated the possibility of profiling metabolite consequences related to seizure activities of an intrinsically low amount of body fluid using CE-MS. It would be useful to investigate and validate, in the future, the known and unknown metabolites in different animal models as well as in humans.
\end{abstract}

\section{Introduction}

Metabolomics has emerged as a valuable tool for diagnostic purposes, to predict and monitor disease progression and to measure therapy efficacy. Moreover, biomarker knowledge provides a deeper understanding of ongoing disease mechanisms, to advance drug discovery and development. Most metabolomic studies were executed by analyzing changes in a single biological sample type, such as tissue, urine, serum or plasma [1,2]. In this study, plasma metabolites were investigated to gain improved knowledge on the metabolic consequences of refractory seizures evoked in the acute $6 \mathrm{~Hz}$ corneal model. An epileptic seizure is defined by the International League Against Epilepsy (ILAE) as a transient occurrence of signs and/or symptoms due to abnormal excessive or synchronous neuronal activity

\footnotetext{
* Corresponding author.

E-mail addresses: karseger@vub.be (K. Segers), w.zhang@lacdr.leidenuniv.nl (W. Zhang), Debby.Mangelings@vub.be (D. Mangelings), hankemeier@lacdr.leidenuniv.nl (T. Hankemeier), Dimitri.De.Bundel@vub.be (D. De Bundel), yvanvdh@vub.be (Y. Vander Heyden), Ilse.Smolders@vub.be (I. Smolders),r.ramautar@lacdr.leidenuniv.nl (R. Ramautar), aveeckha@vub.be (A. Van Eeckhaut).

\# Authors contributed equally to this work.

${ }^{1}$ Equally contributing last authors.
} 


$\begin{array}{ll}\text { Abbreviations } \\ \text { ASD } & \text { anti-seizure drugs } \\ \text { BGE } & \text { background electrolyte } \\ \text { CE } & \text { capillary electrophoresis } \\ \text { CE-MS } & \text { capillary electrophoresis mass spectrometry } \\ \text { FC } & \text { fold change } \\ \text { GABA } & \text { gamma-aminobutyric acid } \\ \text { ILAE } & \text { International League Against Epilepsy } \\ \text { IS } & \text { internal standard } \\ \text { KEGG } & \text { Kyoto Encyclopedia of Genes and Genomes }\end{array}$

in the brain [3]. A disturbance in the balance between excitation and inhibition in the brain indeed results in seizures. It is known that these seizures will affect the metabolism [4]. Increased knowledge about global metabolic hallmarks related to a seizure will lead to an improved understanding of seizure pathophysiology and can reveal novel pathways to control and prevent seizures.

Metabolomics has already been used to investigate changes in the metabolome after seizures [5] or the effect of anti-seizure drug treatment [6] in patients. Most studies, however, focus on brain metabolites by studying brain tissue of a chemo convulsive animal model [7-12]. One of these studies focuses on the participation of cysteine during a seizure and treatment of epilepsy in a mouse model [7]. Another study analyzed different brain regions by NMR spectroscopy, where they found an overlap in changes of the metabolites lactate, succinate, $\gamma$ aminobutyric acid (GABA), choline and taurine in hippocampal tissue and cerebellum of rats [12]. A similar study identified 26 metabolites which are differentiating between the normal group and the rats with a status epilepticus [9]. However, tissue analysis is challenging because of several analytical and technical issues [13]. Therefore, circulating blood-based biomarkers are potentially interesting as plasma is more easily accessible than, for instance, cerebrospinal fluid $[10,14,15]$. At the moment, only a few studies make use of plasma metabolite profiling in epilepsy by using an animal model. As for example the study of Heischmann et al. [8], makes use of a kainic acid model of temporal lobe epilepsy to compare the metabolite depletions found in hippocampal tissue with the observations in plasma. Only a small number of similar metabolic consequences were observed in both matrices. The major change in plasma, $48 \mathrm{~h}$ after a seizure, occurred for triacylglycerols, diacylglycerols, phosphatidylcholines, vitamin D and their derivatives.

Besides animal models, the metabolic consequences of epilepsy are also studied in human samples. One example is the study of Detour et al. [16], which analyzed hippocampal tissue of different patients with temporal lobe epilepsy and divided the samples into groups based on classification criteria by ILAE. Concentration variations of glutamine, glutamate, aspartate, taurine and $\mathrm{N}$-acetylaspartate were observed in the hippocampus of the drug-resistant epilepsy patients. Besides tissue, characterization of drug-resistant epilepsy was conducted in serum samples. Increased values of 3-OH-butyrate, 2-OH-valerate, 2OH- butyrate, acetoacetate, acetone, acetate, choline, alanine, glutamate and scyllo-inositol were observed in patients with epilepsy. Furthermore, decreased levels of glucose, lactate and citrate were observed in these patients [17]. Contradictory, in a study by Al Zweiri et al. [18], which was conducted a few years earlier than the aforementioned study, no prominent markers of responsiveness to drug treatment in epilepsy could be found in serum. Comparable increased levels of glutamate and decreased levels of citrate were observed, based on GC-MS metabolomics [5], as obtained in the study of Murgia et al. [17]. Other findings of this study were the elevated levels of lactate, glutamate and butanoic acid and a reduction of cysteine, glutamine, palmitic acid,

\section{LOF lack-of-fit}

MCR-ALS multivariate curve resolution alternating least squares

NMR nuclear magnetic resonance

NO nitric oxide

PCA principal component analysis

PLS-DA partial least squares discriminant analysis

QC quality control

ROI region of interest

SVD single value decomposition

VIP variable importance in projection linoleic acid, elaidic acid, trans-13-octadecnoic acid, stearic acid, asparagine and glyceraldehyde [5].

Compared to studies in patients, only a few animal studies make use of blood to obtain metabolic profiles in search for markers relevant to epilepsy. As a result of the increased importance of animal welfare (refinement, reduction and replacement), it is not allowed to take more than $20 \mu \mathrm{L}$ mice blood in a serial sampling strategy [19-21]. Alternative sampling techniques, such as volumetric absorptive microsampling $[21,22]$, dried blood spots $[19,21,23]$ and capillary microsampling $[19,21]$ were developed for that purpose. Capillary microsampling has some benefits compared to other microsampling techniques, such as a shorter handling time of the animals, while the equipment for sampling is less expensive than for dried blood spots [19]. In different pharmacokinetic studies, the capillary microsampling technique has already been applied, resulting in robust and reliable outcomes [24-26]. However, these microsampling methods lead to the additional challenge of extracting useful information from small sample volumes. Consequently, the interest in microscale analytical methods has increased in recent years [27,28].

Capillary electrophoresis mass spectrometry (CE-MS) is an interesting microscale technique suitable to profile polar and charged metabolites in small sample volumes [2,27-29]. The relatively low number of applications of CE-MS in biomarker discovery is related to reproducibility and sensitivity issues, as well as the complexity of coupling CE to MS [27]. However, in recent years the CE-MS interface has substantially improved, resulting in an increased sensitivity [30-32]. Several studies have already shown the reliability and repeatability of CE-MS for biomarker discovery [33,34]. Harada et al. [33] performed a large scale cohort study, which profiled more than 8000 human plasma samples with a coefficient of variation for peak areas below $30 \%$ for 80 metabolites. A more recent study of our research group [34], demonstrated the suitability of CE-MS for plasma metabolomics by a simulated omics study combined with two data-analysis tools to handle the data. Drouin et al. [35] proposed the use of the effective mobility of compounds, instead of the migration time, as a more robust migration index. Their work resulted in a database with the effective mobilities of compounds, which will enable a straightforward annotation of detected features in a biological sample.

In the presented study, using an acute $6 \mathrm{~Hz}$ corneal mouse model for refractory seizures, plasma metabolic consequences for seizures were observed in a CE-MS profile through multivariate data analysis. Plasma samples were collected using capillary microsampling at four time points, i.e. before and after the seizure, with a wash-out period of 13 days between the two evoked seizures. All samples were analyzed with a CE-MS method previously developed in the laboratory [34]. Data analysis was conducted by multivariate curve resolution alternating least squares (MCR-ALS) as feature-selection technique, followed by unsupervised and supervised analyses to indicate some possible biomarkers for the evoked seizures. 


\section{Material and methods}

\subsection{Acute $6 \mathrm{~Hz}$ corneal seizure mouse model}

All procedures were carried out according to the National Rules of Animal Experiments (Directive 2010/63) and were approved by the Ethical Committee for Animal Experiments of the Faculty of Medicine and Pharmacy of the Vrije Universiteit Brussel, Belgium (no. 18-213-2). Male NMRI mice, weighing between 38 and $50 \mathrm{~g}$, were obtained from Charles River Laboratories (Sulzfield, Germany) and habituated at least one week at the animal house prior to the experiments. Animals were kept under standard laboratory conditions with a 10/14 h dark/light cycle, with water and food ad libitum. The night before the experiments, the animals were transferred to the experimental room.

A $6 \mathrm{~Hz}$ seizure model as described and applied by [36-39], was used. Corneal stimulation $(6 \mathrm{~Hz}, 0.2 \mathrm{~ms}$ rectangle pulse width, $3 \mathrm{~s}$ duration) by using corneal electrodes connected to a stimulator (ECT Unit 57,800, Ugo-Basile, Italy), induces limbic seizures. The optimal current intensity was determined in 6 animals that showed a seizure duration of more than $20 \mathrm{~s}$ and was found to be $52 \mathrm{~mA}$. Local anesthesia was obtained by applying $0.5 \%$ xylocaine (AstraZeneca, Brussels, Belgium) in saline on the eyes before stimulating. These eye drops additionally result in good conductivity for the electrical stimulation. After stimulation, the animals were placed in an open plastic box to observe the presence or absence of a seizure. Seizures were characterized by a stunned or fixed posture, forelimb clonus and elevated Straub-tail. The seizure durations were manually recorded for each animal.

\subsection{Sample collection}

Blood samples of all animals $(n=8)$ were obtained with capillary microsampling before and after each seizure on the first and the second experimental day. In between both experimental days, a 13-day washout period was applied. In this period the animals were kept under standard laboratory conditions, as aforementioned, and without manipulations. On the first experimental day, the animals received an injection of saline/propylene glycol (50/50 (V/V)) 15 min before the corneal stimulation. On the second experimental day, the animals were injected with a saline solution, $45 \mathrm{~min}$ before the corneal stimulation. The difference in vehicle and time point of injection before the corneal stimulation between the two days of experiments is due to the solubility and half-life time of the administered drug in the treated group. On the first day of experiments the treated group received carbamazepine and on the second experimental day the animals received levetiracetam. However, the samples of the treated groups were not analyzed, as the animals were found not to be drug-resistant (i.e. there were not enough mice that lacked anticonvulsant effects of carbamazepine and levetiracetam), although this was the primary research goal. Therefore, we only included the non-treated animals and solely looked at the metabolic consequences of the provoked seizure. An illustration of the animal experiments with blood sampling time points (1-4) is shown in Fig. 1. Blood was collected using capillary microsampling from the vena saphena [24]. Additional blood was taken from the heart when the animals were sacrificed after the second experimental day. All blood samples were collected into EDTA-coated $32 \mu \mathrm{L}$ capillaries (Virtex Medical, Herlev, Denmark) and plugged 3 times with wax (sealing wax plate, Hirschmann, Eberstadt, Germany). The waxed capillaries were transferred to individually pre-labeled $5 \mathrm{~mL}$ Falcon ${ }^{\circledR}$ tubes (Corning, Tewksbury, Massachusetts, United States) on ice prior to centrifugation. The capillaries were centrifuged in a precooled centrifuge $\left(4{ }^{\circ} \mathrm{C}\right)$ for $10 \mathrm{~min}$ at $1500 \mathrm{~g}$. After centrifugation the capillaries were snapped above the cell layer plug and exactly $10 \mu \mathrm{L}$ of plasma was end-to-end transferred to $10 \mu \mathrm{L}$ capillaries (Virtex Medical, Herlev, Denmark) and stored in labeled Cryo. S vials (Greiner Bio-One, Frickenhausen, Germany). Blood from the heart was collected into EDTA coated tubes (Vacutest, Azergrande, Italy) and centrifuged as previously explained. The plasma layer was transferred to a labeled Eppendorf ${ }^{\circledR}$ tube (Eppendorf, Hamburg, Germany). All plasma samples were stored at $-80{ }^{\circ} \mathrm{C}$ till sample preparation.

\subsection{Quality control samples}

The heart plasma samples of all animals were pooled, $10 \mu \mathrm{L}$ of these pooled samples was transferred into capillaries in Cryo. $S$ vials (Greiner Bio-One, Frickenhausen, Germany) and pretreated as the other plasma samples of the four different time points.

The day of the analysis, samples were reconstituted in $25 \mu \mathrm{L}$ water. From each sample (time point 1-4 and the aliquots of the pooled heart samples), $5 \mu \mathrm{L}$ was taken to generate a pool of them, thereby obtaining quality control (QC) samples to check for and to evaluate system variations. The remaining $20 \mu \mathrm{L}$ of the samples (time point $1-4$ and the aliquots of the pooled heart samples) was used for CE-MS analysis.

The suitability of the CE-MS method for human plasma samples was already demonstrated in [34], but the composition and volume of human plasma is different than that of mice. In order to investigate the effect of capillary microsampling on the sample preparation, $10 \mu \mathrm{L}$

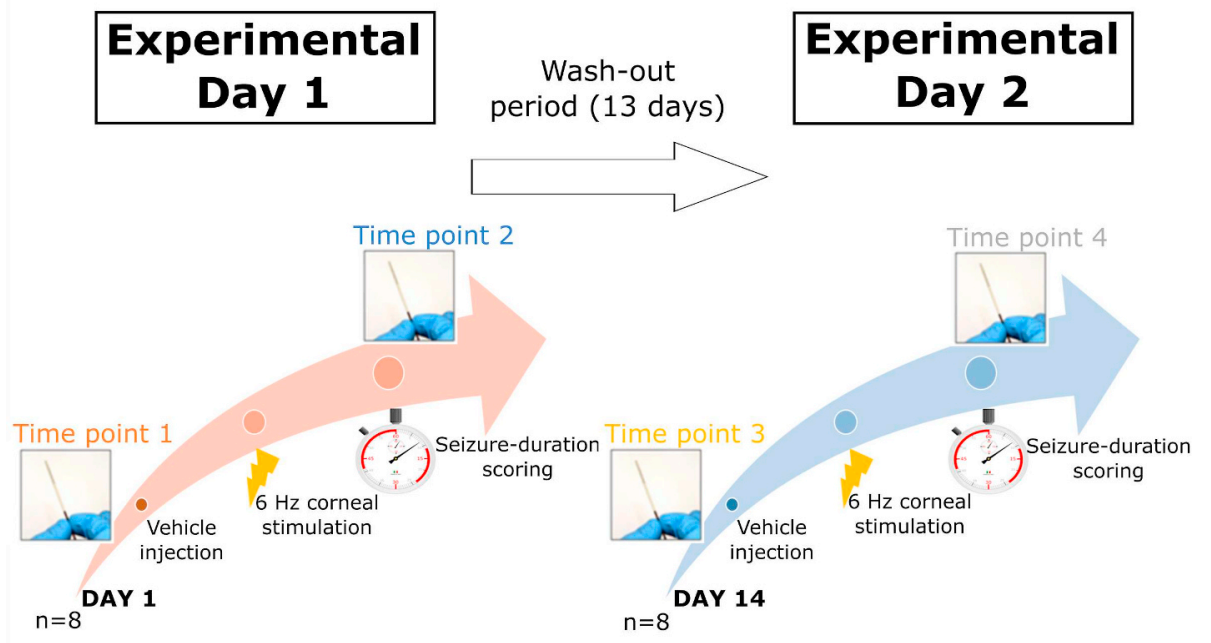

Fig. 1. Illustration of the experimental protocol. On the first day, blood samples were collected before given a vehicle to the animals (time point 1 ). Later, a $6 \mathrm{~Hz}$ stimulation is given, followed by scoring the seizure duration. After the seizure, a second blood sample was collected (time point 2). After a 13-days wash-out period the same experiment was repeated (time point 3 and 4). In total, 4 blood samples were collected: 1 before and 1 after the provoked seizure on both experimental days $(\mathrm{n}=8)$. 
mouse plasma samples was spiked with four labeled compounds (i.e. LAlanine- ${ }^{13} \mathrm{C} 2$, L-Glutamine- ${ }^{13} \mathrm{C} 2$, L-Lysine- ${ }^{13} \mathrm{C} 6$, and L-Isoleucine $-{ }^{13} \mathrm{C},{ }^{15} \mathrm{~N}$ ) (Cambridge Isotope Laboratories, Apeldoorn, The Netherlands) at a series of concentrations based on the naturally existing endogenous levels [40]. Moreover, the samples spiked with intermediate concentration levels were prepared in 8 replicates to monitor the variation in the peak area (precision) and estimated concentration (accuracy).

\subsection{Sample pretreatment}

Plasma samples were prepared as described in [34]. To $10 \mu \mathrm{L}$ stored plasma samples, $250 \mu \mathrm{L}$ Milli-Q water (Millipore, Amsterdam-Zuidoost, the Netherlands), $250 \mu \mathrm{L}$ methanol (HPLC grade, Actu-All Chemicals, Oss, the Netherlands), $250 \mu \mathrm{L}$ chloroform (HPLC grade, Biosolve Chemicals, Valkensweerd, the Netherlands) and $20 \mu \mathrm{L} 30 \mu \mathrm{M}$ internal standard (IS, prepared in Milli-Q water), i.e. DL-methionine sulfone (Human Metabolome Technologies, Leiden, The Netherlands), were added and vortexed followed by centrifugation at $2196 \mathrm{~g}$ at $4{ }^{\circ} \mathrm{C}$ for $10 \mathrm{~min}$. The supernatant $(500 \mu \mathrm{L})$ was transferred to centrifugal filters of $5 \mathrm{kDa}$ (Millipore) and centrifuged for $1.5 \mathrm{~h}$ at $12000 \mathrm{~g} 300 \mu \mathrm{L}$ of the filtrate was transferred after centrifugation and evaporated under vacuum in a CentriVap Concentrator (Labconco). The dried residues were stored at $-80{ }^{\circ} \mathrm{C}$ prior to analysis.

\subsection{Metabolic profiling by $C E-M S$}

The same instruments and analytical methods as in [34] were used. The analysis was conducted on an Agilent 7100 CE instrument hyphenated to an Agilent 6230 Time of Flight mass spectrometer (Agilent Technologies, Santa Clara, California) with a co-axial sheath-liquid interface for the ESI source. The sheath liquid was a mixture of isopropanol/water $(1: 1, \mathrm{~V} / \mathrm{V})$, containing $0.2 \%$ acetic acid and was delivered at $5 \mu \mathrm{L} / \mathrm{min}$. Separation was performed in a fused-silica capillary (BGB Analytik (Harderwijk, The Netherlands)) of $70 \mathrm{~cm}$ length and an internal diameter of $50 \mu \mathrm{m}$, which was thermostated at $22{ }^{\circ} \mathrm{C}$. Milli-Q water containing $10 \%(\mathrm{~V} / \mathrm{V})$ acetic acid (99-100\%) (VWR, Amsterdam, The Netherlands) was used as background electrolyte (BGE). The samples were stored in the sample tray at $10{ }^{\circ} \mathrm{C}$ and injected hydrodynamically at 50 mbar for 20 s. Electrophoretic separation was achieved by applying a voltage of $30 \mathrm{kV}$ and the MS detection was done in positive mode. The MS parameter drying gas flow rate was set at $11 \mathrm{~L} / \mathrm{min}$ and the temperature was $100{ }^{\circ} \mathrm{C}$. Additionally, the nebulizer gas was set at 0 psi. Full scan acquisition, covering the mass range of $50-1000 \mathrm{~m} / z$, was conducted at 1.5 spectra $\mathrm{s}^{-1}$ acquisition rate in centroid mode. Between analyses, the capillary was rinsed as follows: water for $30 \mathrm{~s}$ at $5 \mathrm{bar}$, methanol for $1 \mathrm{~min}$ at $5 \mathrm{bar}$, water for $30 \mathrm{~s}$ at $5 \mathrm{bar}, 10 \%$ ammonium hydroxide for $1 \mathrm{~min}$ at $5 \mathrm{bar}$, water for $30 \mathrm{~s}$ at 5 bar and BGE for $2 \mathrm{~min}$ at 5 bar.

\subsection{Data processing}

The obtained data were stored as. $\mathrm{d}$ files and converted to mzXML formats with ProteoWizard (Palo Alto, California). These files were imported and further analyzed in MatlabTM R2014a (The Mathworks, Natick, MA), as described in [34].

First, a binning method was performed and data were compressed through the detection of regions of interest (ROI) [41,42]. ROI values were searched for each migration time in the CE-MS profile. However, each ROI is defined by a signal threshold, mass accuracy and the minimum time interval to be considered as peak width [43,44]. In our study, the parameters are based on the protocol by Gorrochategui et al. [44] and were set at 1000 for the signal threshold, 0.01 atomic mass unites/e was used as mass accuracy and $6 \mathrm{~s}$ as the minimum time interval.
After the ROI search in the metabolic profiles, feature detection was performed with the MCR-ALS toolbox [45]. MCR-ALS does not require peak alignment, which makes it very suitable for CE data, where significant migration time shifts are typically observed [43]. The number of components in the samples is initially guessed. The Single Value Decomposition (SVD) method is the most suitable to resolve features. Different SVD-numbers in an MCR-ALS model were evaluated, considering the explained variance and the lack-of-fit (LOF) error of the model. To detect the purest variables, a $10 \%$ noise level threshold was used. The features were detected by the best MCR-ALS model, containing the highest explained variance and the lowest LOF error, were then further investigated.

Considering that the peak area calculation by MCR-ALS was not ideal, the obtained features were further investigated in the Data Acquisition module within the MassHunter Workstation (Agilent, Santa Clara, CA), which results in a proper integration of the detected features. The result is a peak table containing areas of the obtained features in all samples. After integration with the MassHunter Data acquisition module, the peak areas of the integrated features were normalized with that of the internal standard and then auto-scaled prior to further statistical analysis.

\subsubsection{Multivariate analysis}

The normalized and auto-scaled data were visualized with exploratory unsupervised analysis techniques, such as clustering analysis (complete linkage) and principal component analysis (PCA). PLS-DA was performed as supervised classification technique to link the sample time point with the obtained fingerprint. The numbers of latent variables for the models of the supervised analyses were chosen based on a five-fold venetian-blind cross validation. Subsequently, a model was evaluated based on the error rate, non-error rate and accuracy on the cross-validation and calibration results. In the end, possible seizure biomarkers are reported based on a VIP score above 1.0, which reflects the importance of the variable in the discrimination between the groups of interest, i.e. before and after a seizure.

\subsubsection{Univariate analysis and fold change analysis}

A pair-wise comparison was performed on the normalized data to investigate the differences between the time points within and between days, illustrated in Fig. 1. The animals were their own controls, which allowed performing a paired samples test. Since the normal distribution pattern of the differences could not be verified due to the small sample size $(n=8)$, the non-parametric signed-rank or paired-samples Wilcoxon test was used.

Besides the statistical p-value also fold changes (FC) are calculated to demonstrate the strength of the increase $(>1)$ or decrease $(<1)$ of a metabolite in plasma. Those FC values can be compared between both evoked seizures. Additionally, the effectiveness of the wash-out period can be shown by a FC of approximately 1 . FC are calculated for the comparison of time point 2 with $1\left(\mathrm{FC}_{2 / 1}\right)$, time point 4 with $3\left(\mathrm{FC}_{4 / 3}\right)$ and time point 3 with $1\left(\mathrm{FC}_{3 / 1}\right)$. The calculations are performed as shown in equations (1)-(3).

$$
\begin{aligned}
& F C_{2 / 1}=\frac{\text { Ratio } A U C / A U C_{\text {IStime point } 2}}{\text { Ratio AUC/AUC IStime point } 1} \\
& F C_{4 / 3}=\frac{\text { Ratio } A U C / A U C_{\text {IStime point } 4}}{\text { Ratio AUC/AUC } C_{\text {IStime point } 3}} \\
& F C_{3 / 1}=\frac{\text { Ratio } A U C / A U C_{\text {IStime point } 3}}{\text { Ratio AUC/AUC } \text { IStime point } 1_{1}}
\end{aligned}
$$




\section{Results and discussion}

\subsection{Evaluation of analytical workflow for metabolic profiling of volume- restricted plasma samples}

When performing metabolic profiling of volume-restricted samples, aspects like sampling, sample preparation and analysis by CE-MS need to be carefully evaluated in order to minimize technical variations. Therefore, we have first assessed whether the plasma sampling technique had an effect on the CE-MS results. For this purpose, plasma samples $(n=8)$ were spiked with four labeled compounds at concentrations reflecting the endogenous levels of the natural counterparts in plasma. Those samples were then stored in $10 \mu \mathrm{L}$ capillaries, as the actual samples. The relative standard deviations (RSD) obtained for the peak areas of these compounds were below $7.4 \%$. The accuracy was also investigated by comparing the spiked with the experimental concentrations. The results for the accuracy test fall in an acceptable range, i.e. between 85 and $115 \%$ (data not shown).

Before starting the main analysis, one QC sample was analyzed ten times to examine the repeatability of the CE-MS method. After obtaining a signal, repeatable in profile and intensity, the actual samples were analyzed randomly and alternated with blank solutions and QC samples (total number of QC samples $=13$ ) and at the end some metabolite standards (including the following compounds: glycine, L-alanine, cytosine, proline, valine, homoserine, threonine, creatine, isoleucine, leucine, asparagine, adenine, anthranilic acid, tyramine, spermidine, glutamine, lysine, glutamic acid, methionine, histidine, phenylalanine, spermine, tryptophan, cytidine, adenosine), which can be used for identification. The analysis of the QC samples will reflect the system variation. As described in literature, to be reliable, RSD values for the resolved features have to be below $30 \%$ for the AUC values and below $5 \%$ for the migration times [46]. In this study, 44 measured $m / z$ values in the QC samples showed acceptable RSD values (below $20.3 \%$ for the corrected areas and below $2.5 \%$ for the migration times). Two representative extracted ion electropherograms for the reliable features (44) detected in a QC sample are shown in Fig. 2. A drift correction was not performed because the instrumental drift was negligible compared to the biological variation.

The variation in sample preparation was verified with the pooled heart plasma sample, which was divided in $10 \mu \mathrm{L}$ aliquots undergoing the sample pretreatment. The RSD for all features taken into account measured in the heart plasma samples were below $19.5 \%$ and $0.7 \%$ for the corrected areas and migration times, respectively. The repeatability of injection was examined by injecting the samples of the fourth time point twice, resulting in RSD values between the two injections below $20.2 \%$ and $3.5 \%$ for the corrected areas and migration times, respectively.

Summarized, all estimated variations were within the acceptance limits, which demonstrates the reliability of the CE-MS method and

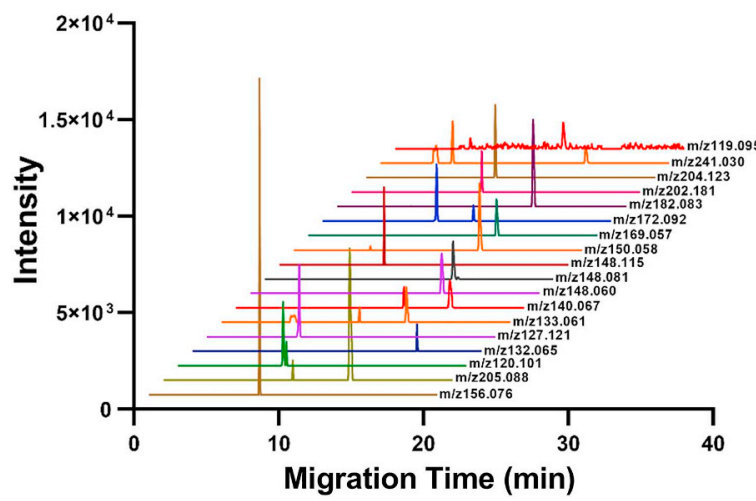

sampling technique to investigate the metabolites in volume-restricted plasma samples.

\subsection{Metabolic profiling of plasma samples from a mouse model for epileptic seizures}

The focus of this paper is to investigate the applicability of the previously mentioned CE-MS method to detect plasma metabolites related to metabolic consequences for an evoked seizure. In total, 74 samples were analyzed, including 32 from the epileptic mouse model, 8 mouse heart samples, 8 samples from the $4^{\text {th }}$ time point injected a second time, 7 blank solutions, 6 standards, and 13 quality controls which were injected between the different groups ad random. Data compression resulted in 161 resolved $m / z$ values. All $m / z$ values were manually evaluated to exclude spikes. Spikes can be the result of electrical disturbance occurring in the MS system and are not related to a metabolite. The feature detection allowed to reduce the data to $57 \mathrm{~m} /$ $z$ values with good predictive ability of the resulting MCR-ALS model; i.e. $99.2 \%$ explained variance and $9.2 \%$ LOF error. However, this data set also contains $\mathrm{m} / \mathrm{z}$ values with an RSD above $30 \%$ for their peak area ratios and of more than $5 \%$ for the migration times. After removing these non-compliant features, $44 \mathrm{~m} / \mathrm{z}$ values remained, which have acceptable variations for migration times and corrected peak areas measured for the QC samples, i.e. $2.5 \%$ and $20.3 \%$, respectively. Besides the non-parametric Wilcoxon test and fold change investigations, multivariate analysis was performed on these 44 features after applying auto-scaling to eliminate the dominance of highly abundant features.

First, the sample similarity was investigated by constructing a dendrogram as shown in Fig. 3. This dendrogram was constructed using the complete linkage method and the Euclidean distance as similarity parameter. The further the samples are located from each other on the $\mathrm{X}$-axis, the more dissimilar. Three clusters are clearly revealed in the dendrogram. The left group contains all samples after the first seizure (i.e. Time point 2), illustrating high similarities between these profiles and a clear distinction from all other time points. Notably, sample 23 from Time point 3 (i.e. after the wash-out period) is also present in this cluster. The possible reason for similarity of sample 23 with these cluster profiles may be related to the short seizure duration of the animal (22 s).

Only three samples after the second seizure (Time point 4) are clustering together with samples after the first seizure (Time point 2) in the left cluster, which can be attributed to similar seizure durations for both onsets. The mean durations of the first and second evoked seizures were $3 \mathrm{~min} 2 \mathrm{~s}$ and $1 \mathrm{~min} 23 \mathrm{~s}$, respectively. However, five samples after the second seizure (Time point 4 ) are more similar with Time point 1 and 3. These five animals showed a decreased seizure duration compared to the first seizure, which might result in a profile that is more comparable to these at time point 1 and 3 . From this clustering

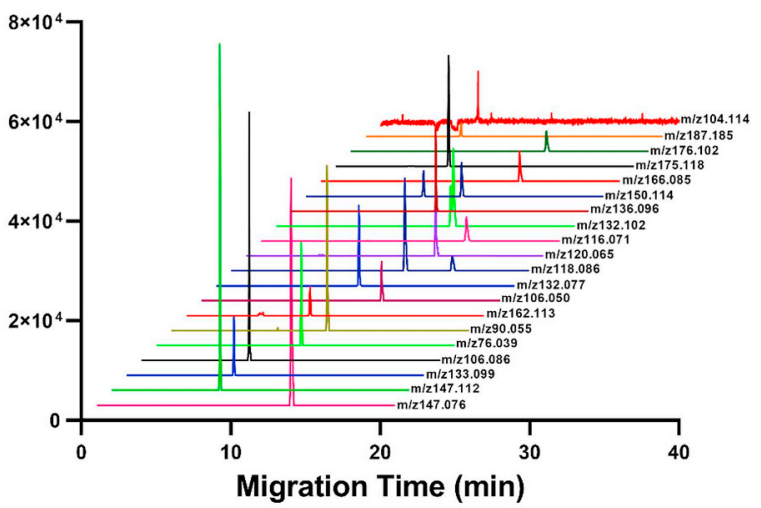

Fig. 2. Reconstructed extracted ion electropherograms of the 44 reliable features $(\mathrm{m} / z$ values) in a QC sample as obtained by CE-MS. 


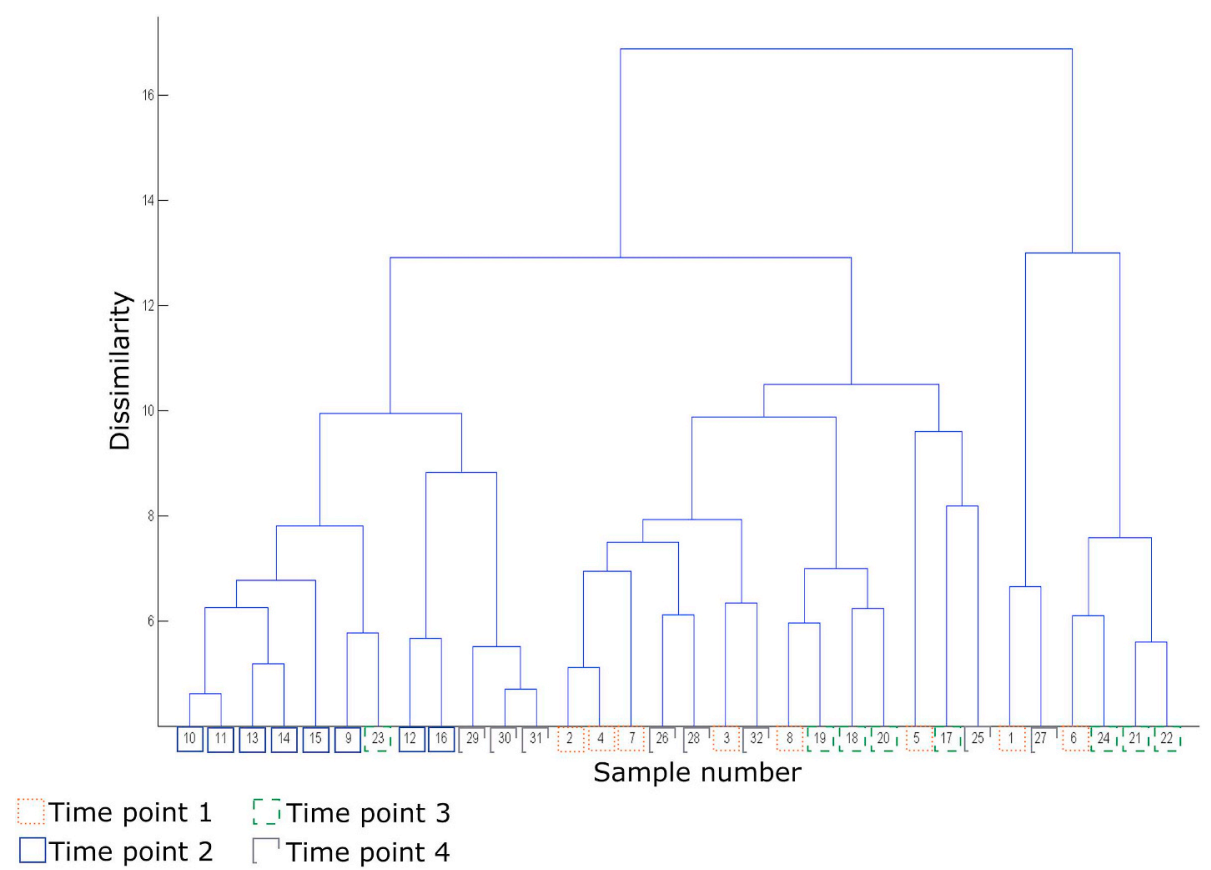

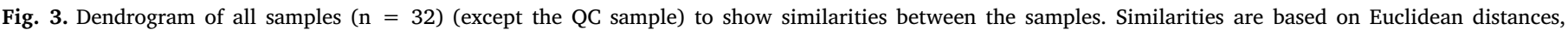
calculated on the autoscaled $\mathbf{X}$ matrix.

information, no clear distinction is observed between the first and thirdtime point profiles, suggesting the effectiveness of the wash-out period. The analogies at different time points and the related markers are studied in more detail by PCA and in the statistical analysis.

The main objective was to find metabolic consequences of evoked seizures in plasma. For that purpose profiles at time points 1 and 2, and at time points 3 and 4 were compared. The overall clustering can be seen in Fig. 4A. Here, a clear discrimination between the first sampling time point in the naïve mice with the sample after a seizure can be observed. Clustering tendency of the samples before and after a seizure on the two experimental days is shown in PCA score plots (Fig. 4B and C). For the first experimental day, the samples before a seizure (represented by black dots in Fig. 4B) are clearly discriminated from those after a seizure (represented by pink squares in Fig. 4B), suggesting a dissimilar profile and changes that occurred by an evoked seizure. The assessment on VIP scores led to the indication of 21 potential markers, which were all statistically significant $(\mathrm{p}<0.05)$ by univariate statistics (non-parametric paired-samples Wilcoxon test). The VIP scores resulted from a PLS-DA model with 1 latent variable having good predictive abilities $(100 \%$ accuracy and non-error rate for both calibration and cross-validation). Only 17 markers, shown in Table 1, could be identified with an in-house standard mixture of Drouin et al. [35], containing more than 450 compounds, and by applying the ROMANCE software to calculate the effective electrophoretic mobilities [47]. This annotation resulted in identified metabolites with the annotation of at least two independent and orthogonal properties, i.e. accurate mass and electrophoretic mobility. Features, which could not be annotated by the in-house library, were annotated by the METLIN database [48]. This approach resulted in too many metabolite options with a high level of uncertainty and therefore these annotations were not included in the table. In general, mass errors were below $16 \mathrm{ppm}$, which is acceptable as the acceptance limit was set at $20 \mathrm{ppm}$ [49]. Only one identified metabolite, i.e. tryptophan (Table 3), has a mass error of $23.2 \mathrm{ppm}$ and the mobility variation is also higher than the acceptance limit of $10 \%$ [35].

All metabolites in Table 1 were decreased in concentration after a seizure, as demonstrated by Fig. 5, which contains concentration trends and FC ratios of some identified amino acids of Table 1. Decreased concentrations of isoleucine, serine, citrulline, histidine and proline after a seizure are demonstrated by their FC $<1$ and are also observed in the study of Heischmann et al. [8]. These authors investigated metabolite changes in rat plasma, $48 \mathrm{~h}$ after a seizure occurred in a chemo convulsive model for seizures. The obtained FC ratios in our study are similar to the ranges reported by Wang et al. [5] and Tan et al. [9] and will indicate, when $p<0.05$ and VIP-scores $>1$, metabolites that change due to metabolic consequences of a seizure.

When comparing the data files obtained before (represented by green triangles in Fig. 4C) and after (represented by red diamonds in Fig. 4C) the second evoked seizure, less dissimilarity is seen between the samples after the 13-days wash-out period. This reduced dissimilarity may be the result of the injected vehicle, which is different between the first (propylene glycol/saline $(50 / 50 \mathrm{~V} / \mathrm{V})$ ) and second experimental (saline) day, or due to the possibility that a second evoked seizure will induce less metabolic consequences in plasma than a first. However, this last consequence is in contradiction with "seizures beget seizures", but epilepsy and seizures are brain-specific disorders and many compounds do not cross the blood-brain barrier [8]. Another explanation could be related to the duration of the effects by an evoked seizure. Therefore, this result may suggest the prolonged effect of a first seizure reflected by the metabolite changes. The PLS-DA model resulted in an accuracy of $88 \%$ for calibration and $63 \%$ for cross-validation results, suggesting less good predictive abilities of the model compared to the previous PLS-DA model for the first day of experiments. Only four metabolites were found statistically significant based on non-parametric univariate statistics, i.e. glutamate $\left(\mathrm{FC}_{4 / 3}=1.09\right)$, glutamine $\left(\mathrm{FC}_{4 / 3}=1.11\right)$, 4-hydroxyproline $\left(\mathrm{FC}_{4 / 3}=0.87\right)$ and methionine $\left(\mathrm{FC}_{4 /}\right.$ $\left.{ }_{3}=0.88\right)$. The latter two amino acids showed a decrease in plasma concentration $\left(\mathrm{FC}_{4 / 3}<1\right)$ after the seizure, while glutamate and glutamine plasma concentrations were increased $\left(\mathrm{FC}_{4 / 3}>1\right)$ in this study. Glutamate was also increased in human serum samples in the studies of Wang et al. [5] and Murgia et al. [17]. Additionally, in a chemo convulsive rat model increased glutamate levels were also observed in hippocampal tissue [9].

For both evoked seizures $\left(1^{\text {st }}\right.$ and $\left.2^{\text {nd }}\right)$ some metabolites were indicated by VIP-scores above 1 . The amino acids lysine, glycine, phenylalanine, methionine, citrulline and histidine showed for both 

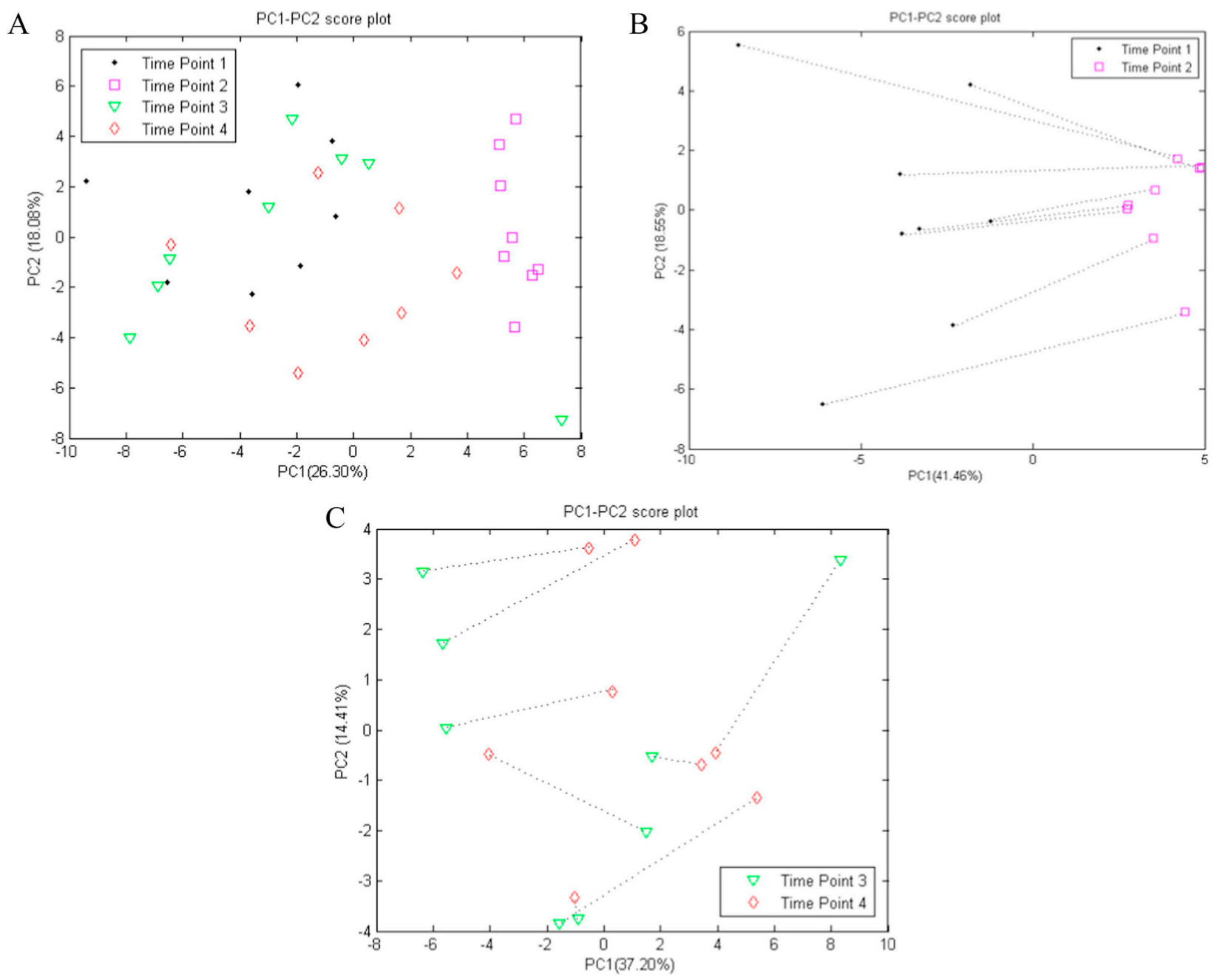

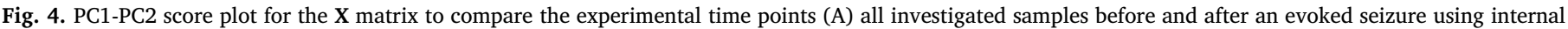

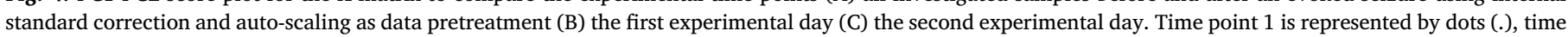
point 2 by squares $(\square)$, time point 3 is represented triangles $(\Delta)$ and time point 4 by diamonds $(\diamond)$.

seizures a decrease in plasma concentrations $\left(\mathrm{FC}_{2 / 1}\right.$ and $\left.\mathrm{FC}_{4 / 3}<1\right)$, as shown in Fig. 5. The less pronounced decrease for a second seizure is additionally reflected by the higher value of the $\mathrm{FC}_{4 / 3}$ ratio on the second day for the aforementioned metabolites compared to the $\mathrm{FC}_{2 / 1}$ ratio for the first seizure. A higher value for the FC ratio (which is still below 1) means a lower decrease in plasma levels. For example, lysine has on the first day a $\mathrm{FC}_{2 / 1}$ of 0.69 and for the second day the $\mathrm{FC}_{4 / 3}$ was 0.88 . For the other amino acids, glycine, phenylalanine, methionine, citrulline and histidine, the $\mathrm{FC}_{4 / 3}$ for the second seizure are $0.90,0.90$, $0.88,0.85$ and 0.92 , respectively. From the univariate paired-samples Wilcoxon-test the levels of all aforementioned amino acids are significantly decreased $(\mathrm{p}<0.05)$ for the first seizure, but after the second seizure, significance is only obtained for methionine.

The decreased plasma concentration of proline after a seizure in the $6 \mathrm{~Hz}$ mouse model is in contrast with the serum concentrations reported in Ref. [5], which compared control groups with patients who had a seizure within $48 \mathrm{~h}$ before sampling.

The lack of effectiveness of the wash-out period could be another explanation to the less pronounced metabolic changes for a second seizure. However, the similarities between time point 1 and 3 suggested an effectiveness of the wash-out period. This similarity was demonstrated by their clustering pattern in the PCA score plot (not shown). Still, multivariate analyses revealed some responsible markers for the distinction between the first seizure (Time point 2) and the post washout period profiles (Time point 3), which are listed in Table 2.
Noteworthy, univariate results revealed that most (but not all) metabolites with a VIP score above 1.0 were statistically different after the wash-out period. Therefore, proper selection of a threshold for the VIP score is important when using this parameter [50]. The threshold is accordingly (study specific) often adjusted to values above 1 [51,52]. Thus, the VIP score allows to indicate potential biomarker candidates without defining it as a biomarker [50]. In our study, the plasma levels of glycine, serine, asparagine, threonine, methionine, alanine, 3-methyladenine, citrulline, arginine, histidine, ornithine and lysine were increased (13 days after the first seizure) compared to their post seizure values. These increased values after the wash-out demonstrates the influence of sampling time after a seizure, which may result in different interpretations. Additionally, these increased levels, 13 days after the seizure, reflect also the stabilization of the plasma levels during the wash-out period. This stabilization is demonstrated by the concentration trends of some metabolites shown in Fig. 5 and by a $\mathrm{FC}_{3 / 1}$ of approximately 1 , indicating that most metabolites are present at similar concentration levels at both time points 1 and 3 .

Another interesting aspect is the re-use of animals in an acute $6 \mathrm{~Hz}$ animal model. However, to our knowledge, nothing is currently known about the similarity of the metabolite profiles over the different seizures. Therefore, the comparison between the second and fourth time point is made in Fig. 6. A clear discrimination of the samples belonging to the first and second evoked seizure is demonstrated by this score plot, suggesting a difference in metabolic consequences observed in 
Table 1

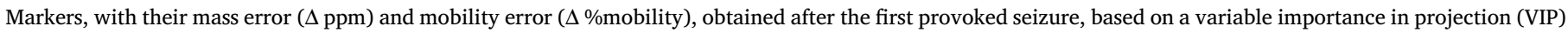
score above 1 and p-value below 0.05 .

\begin{tabular}{|c|c|c|c|c|c|c|c|}
\hline Compound & {$[\mathrm{M}+\mathrm{H}]^{+}$} & $\Delta \mathrm{ppm}$ & Migration time (min) & $\Delta \%$ mobility & p-value ${ }^{b}$ & VIP score & Plasma concentration $^{c}$ \\
\hline Methionine & 150.058 & 6.2 & 12.4 & 1 & 0.0078 & 1.5 & Decreased \\
\hline Unknown & 148.115 & - & 7.5 & - & 0.0078 & 1.5 & \\
\hline Lysine & 147.113 & 3.9 & 7.1 & 0 & 0.0078 & 1.5 & \\
\hline Glycine & 76.040 & 6.1 & 9.4 & 0 & 0.0078 & 1.5 & \\
\hline Alanine & 90.055 & 3.2 & 10.1 & 0 & 0.0078 & 1.4 & \\
\hline Unknown & 136.096 & - & 9.5 & - & 0.0078 & 1.4 & \\
\hline Arginine & 175.118 & 1.3 & 7.4 & 2 & 0.0078 & 1.3 & \\
\hline Threonine & 120.065 & 4.2 & 12.2 & 1 & 0.0078 & 1.3 & \\
\hline 3-methyladenine & 150.111 & 15.8 & 12.5 & 7 & 0.0234 & 1.3 & \\
\hline Unknown & 176.121 & - & 7.5 & - & 0.0006 & 1.3 & \\
\hline Leucine/Isoleucine $^{\text {a }}$ & 132.101 & 5.2 & 11.5 & 0 & 0.0078 & 1.3 & \\
\hline Serine & 106.050 & 4.9 & 11.7 & 0 & 0.0078 & 1.3 & \\
\hline Asparagine & 133.060 & 8.7 & 12.3 & 1 & 0.0078 & 1.2 & \\
\hline Citrulline & 176.102 & 4.2 & 12.6 & 1 & 0.0078 & 1.2 & \\
\hline Unknown & 172.092 & - & 10.5 & - & 0.0078 & 1.2 & \\
\hline Unknown & 119.093 & - & 11.5 & - & 0.0078 & 1.2 & \\
\hline Phenylalanine & 166.085 & 4.5 & 12.8 & 1 & 0.0078 & 1.2 & \\
\hline Ornithine & 133.100 & 1.5 & 7.1 & 1 & 0.0078 & 1.2 & \\
\hline Valine & 118.086 & 6.7 & 11.3 & 1 & 0.0078 & 1.2 & \\
\hline Histidine & 156.076 & 4.3 & 7.5 & 1 & 0.0078 & 1.1 & \\
\hline Proline & 116.070 & 7.5 & 13.2 & 1 & 0.0391 & 1.0 & \\
\hline
\end{tabular}

a Compounds present in the standard mixture, but not baseline separated.

b p-value from a non-parametric signed-rank Wilcoxon test.

c Compared to the basal levels.

plasma for a second evoked seizure compared to a first. VIP score investigation resulted in the suggestion of 20 markers, of which 15 could be identified (see Table 3). However, the identification of tryptophan is not within the limits of mass error (23.2 ppm, where the limit is
$20 \mathrm{ppm}$ ) [49] and of difference in mobility (12.4\%, with limit 10\%) [35]. Although identification parameters are just outside the acceptance limits, tryptophan is further discussed in the biological interpretation part. Higher plasma levels after the second seizure than after
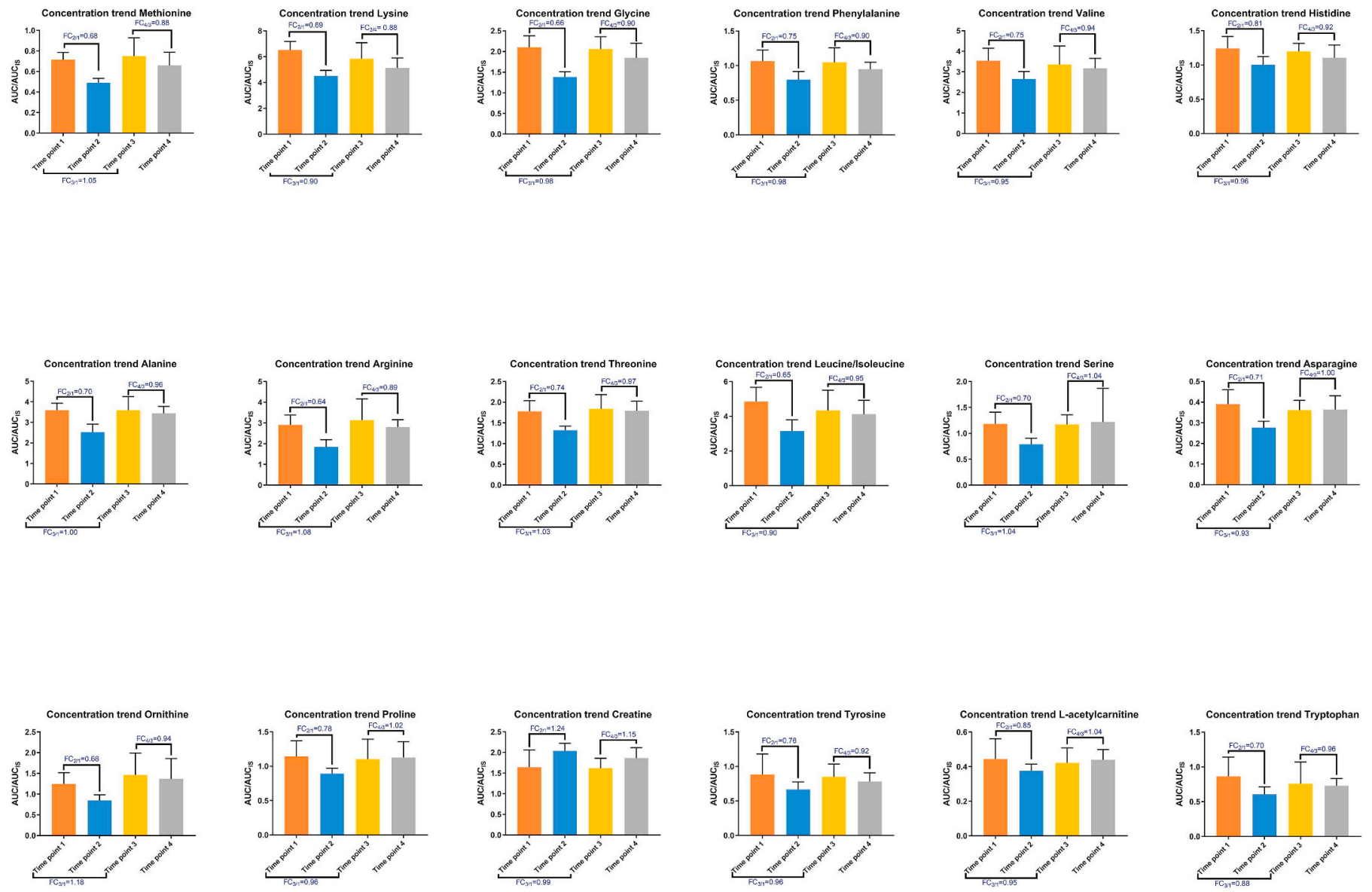

Fig. 5. Concentration trends, between the different investigated time points, of some identified amino acids with their fold change (FC). 
Table 2

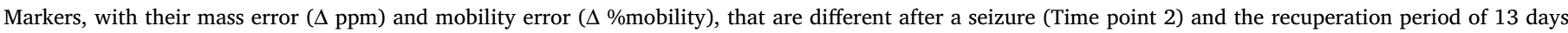
(Time point 3 ), based on a VIP score above 1.

\begin{tabular}{|c|c|c|c|c|c|c|c|}
\hline Compound & {$[\mathrm{M}+\mathrm{H}]^{+}$} & $\Delta \mathrm{ppm}$ & Migration time (min) & $\Delta \%$ mobility & p-value ${ }^{b}$ & VIP score & Plasma concentration ${ }^{c}$ \\
\hline Glycine & 76.040 & 6.1 & 9.4 & 0 & 0.0078 & 1.6 & Increased \\
\hline Unknown & 136.096 & - & 9.5 & - & 0.0078 & 1.6 & \\
\hline Serine & 106.050 & 4.9 & 11.7 & 0 & 0.0078 & 1.5 & \\
\hline Asparagine & 133.060 & 8.7 & 12.3 & 1 & 0.0078 & 1.5 & \\
\hline Threonine & 120.065 & 4.2 & 12.2 & 1 & 0.0078 & 1.4 & \\
\hline Methionine & 150.058 & 6.2 & 12.4 & 1 & 0.0156 & 1.4 & \\
\hline Alanine & 90.055 & 3.2 & 10.1 & 0 & 0.0156 & 1.4 & \\
\hline Creatine & 132.076 & 2.2 & 9.3 & 1 & 0.0078 & 1.4 & Decreased \\
\hline Unknown & 172.092 & - & 10.5 & - & 0.0234 & 1.3 & Increased \\
\hline 3-methyladenine & 150.111 & 15.8 & 7.74 & 7 & 0.0234 & 1.3 & \\
\hline Citrulline & 176.102 & 4.2 & 12.6 & 1 & 0.0156 & 1.3 & \\
\hline Arginine & 175.118 & 1.3 & 7.4 & 2 & 0.0156 & 1.3 & \\
\hline Unknown & 176.121 & - & 7.5 & - & $0.1215^{d}$ & 1.3 & \\
\hline Histidine & 156.076 & 4.3 & 7.5 & 1 & 0.0156 & 1.3 & \\
\hline Ornithine & 133.100 & 1.5 & 7.1 & 1 & 0.0234 & 1.3 & \\
\hline Phenylalanine & 166.085 & 4.5 & 12.8 & 1 & $0.0547^{d}$ & 1.2 & \\
\hline Lysine & 147.113 & 3.9 & 7.1 & 0 & 0.0391 & 1.2 & \\
\hline Unknown & 132.065 & - & 9.5 & - & 0.0078 & 1.2 & Decreased \\
\hline Unknown & 148.115 & - & 7.5 & - & $0.0781^{d}$ & 1.1 & Increased \\
\hline Leucine/Isoleucine $^{\mathrm{a}}$ & 132.101 & 5.2 & 11.5 & 0 & $0.1799^{d}$ & 1.1 & \\
\hline Tyrosine & 182.083 & 10 & 13.0 & 1 & $0.0781^{\mathrm{d}}$ & 1.0 & \\
\hline Unknown & 104.114 & - & 6.5 & - & $0.1094^{d}$ & 1.0 & Decreased \\
\hline Unknown & 119.093 & - & 11.5 & - & $0.1484^{d}$ & 1.0 & Increased \\
\hline
\end{tabular}

a Compounds present in the standard mixture, but not baseline separated.

b p-value from a non-parametric signed-rank Wilcoxon test.

c Changes in plasma concentration between seizure and after the wash-out period (13 days after first seizure).

d Non-significant differences.

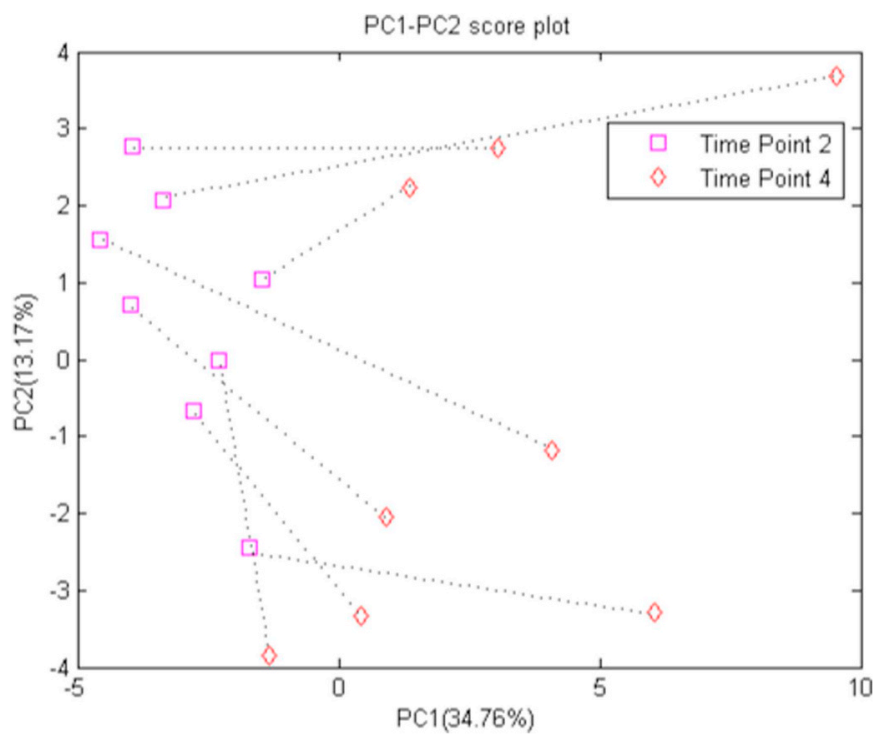

Fig. 6. PC1-PC2 score plot for the $\mathbf{X}$ matrix to compare the profiles after two different seizures using internal standard correction and autoscaling as data pretreatment. Time point 2 is represented by green squares $(\square)$ and time point 4 by red diamonds $(\diamond)$. (For interpretation of the references to colour in this figure legend, the reader is referred to the Web version of this article.)

the first were obtained for arginine, threonine, alanine, methionine, glycine, asparagine, 3-methyladenine and citrulline as shown in Fig. 5. For glycine, methionine and citrulline higher FC for the second day $\left(\mathrm{FC}_{4 / 3}\right)$ were found as already discussed above. The other amino acids, arginine, threonine, alanine, asparagine and 3-methyladenine had a $\mathrm{FC}_{4 / 3}$ of $0.89,0.97,0.96,1.00$ and 0.96 , respectively. All these $\mathrm{FC}_{4 / 3}$ ratios for the second seizure are higher than the equivalent $\mathrm{FC}_{2 / 1}$, which are $0.64,0.74,0.70,0.71$ and 0.74 , shown for some metabolites in Fig. 5. Our results demonstrate that a second seizure with the same stimulation may result in a less pronounced decrease of the metabolite levels in mouse plasma, compared to the decrease observed after the first evoked seizure, which can also be seen in the concentration trend profiles of Fig. 5 and the $\mathrm{FC}$ ratios $\left(\mathrm{FC}_{2 / 1}, \mathrm{FC}_{4 / 3}\right.$ and $\left.\mathrm{FC}_{3 / 1}\right)$.

\subsection{Biological significance of the obtained findings}

Pathway analysis with the Kyoto Encyclopedia of Genes and Genomes (KEGG) software assigned most of the identified compounds to the nucleotide and amino acid metabolism. The identified markers, most of which are amino acids, play important roles in maintaining the equilibrium between inhibitory and excitatory processes.

A seizure is the result of an imbalance between excitation and inhibition. The most important excitatory neurotransmitter is glutamate. Most of the identified compounds in our work are directly or indirectly related to glutamate, which shows elevated levels in our study after a second seizure and in serum and extracellular brain tissues in other publications [53-56]. A precursor of glutamate is glutamine, and a decreased metabolization of glutamate to glutamine causes glutamate accumulation resulting in a seizure [56-58]. This decreased values of glutamine are obtained in plasma samples of a chemo convulsive rat model [8] and in human serum samples [5] and are in contradiction with the increased values observed in our study after a second evoked seizure and in hippocampal sclerosis samples of humans in [16]. Other metabolites indirectly involved in the glutamate-glutamine cycle include alanine, asparagine and leucine $[59,60]$, which are all decreased in our observations after an evoked seizure. This decrease in asparagine is also described by Wang et al. [5] in human serum samples. The decreased plasma level of alanine is also observed in human hippocampal sclerosis tissue [16].

Besides an increase in glutamate, the role of norepinephrine, epinephrine, dopamine and serotonin is not fully clarified. A decrease of these monoamines has been reported in epileptic seizures $[59,61,62]$, but in other scientific reports increased levels are described [63]. Therefore, a decrease in the precursors of those neurotransmitters, such as tryptophan, phenylalanine and tyrosine, is expected after a seizure $[56,60,64]$ and demonstrated by our findings. This decrease of the 
Table 3

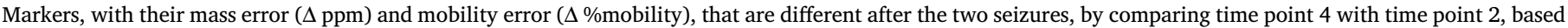
on a VIP score above 1.0.

\begin{tabular}{|c|c|c|c|c|c|c|c|}
\hline Compound & {$[\mathrm{M}+\mathrm{H}]^{+}$} & $\Delta \mathrm{ppm}$ & Migration time (min) & $\Delta \%$ mobility & p-value ${ }^{c}$ & VIP score & Plasma concentration $^{\mathrm{d}}$ \\
\hline Arginine & 175.118 & 1.3 & 7.4 & 2 & 0.0078 & 1.7 & Increased \\
\hline Threonine & 120.065 & 4.2 & 12.2 & 1 & 0.0078 & 1.6 & \\
\hline Alanine & 90.055 & 3.2 & 10.1 & 0 & 0.0078 & 1.6 & \\
\hline Unknown & 176.121 & - & 7.5 & - & $0.0756^{\mathrm{e}}$ & 1.6 & \\
\hline 3-methyladenine & 150.111 & 15.8 & 12.5 & 7 & 0.0078 & 1.5 & \\
\hline Unknown & 172.092 & - & 10.5 & - & 0.0078 & 1.5 & \\
\hline Unknown & 119.093 & - & 11.5 & - & 0.0156 & 1.4 & \\
\hline Methionine & 150.058 & 6.2 & 12.4 & 1 & 0.0078 & 1.4 & \\
\hline Glycine & 76.040 & 6.1 & 9.4 & 0 & 0.0078 & 1.4 & \\
\hline Asparagine & 133.060 & 8.7 & 12.3 & 1 & 0.0078 & 1.3 & \\
\hline Unknown & 136.096 & - & 9.5 & - & 0.0078 & 1.3 & \\
\hline Ornithine & 133.100 & 1.5 & 7.1 & 1 & $0.0781^{\mathrm{e}}$ & 1.2 & \\
\hline Phenylalanine & 166.085 & 4.5 & 12.8 & 1 & $0.0547^{e}$ & 1.2 & \\
\hline Proline & 116.070 & 7.5 & 13.2 & 1 & $0.0547^{\mathrm{e}}$ & 1.2 & \\
\hline Leucine/Isoleucine $^{\mathrm{a}}$ & 132.101 & 5.2 & 11.5 & 0 & $0.0781^{\mathrm{e}}$ & 1.2 & \\
\hline L-acetylcarnitine & 204.123 & 1 & 8.7 & 1 & 0.0391 & 1.1 & \\
\hline Unknown & 104.114 & - & 6.5 & - & 0.0391 & 1.1 & Decreased \\
\hline Valine & 118.086 & 6.7 & 11.3 & 1 & $0.0781^{3}$ & 1.1 & Increased \\
\hline Tryptophan ${ }^{\mathrm{b}}$ & 205.081 & 23.2 & 12.4 & 12 & 0.0391 & 1.1 & \\
\hline Citrulline & 176.102 & 4.2 & 12.6 & 1 & $0.0547^{3}$ & 1.1 & \\
\hline
\end{tabular}

\footnotetext{
a Compounds present in the standard mixture, but not baseline separated.

b Conflict of identification, due to too high mass error and mobility difference.

c p-value from a non-parametric signed-rank Wilcoxon test.

d Changes in plasma concentrations between first and second evoked seizure.

e Non-significant differences.
}

precursor levels can be due to the decrease of the monoamines or due to the overconsumption.

GABA is known as inhibitory neurotransmitter. Decreased GABA levels can be found in neurodegenerative and epileptic disorders, as a result of its use as an alternative energy source, instead of isoleucine and valine [59]. Decreased values of these latter amino acids are observed in our study and the decrease of valine is also described by Detour et al. [16] in hippocampal tissue. Other metabolites, such as glycine, serine, threonine $[56,57,60,65]$, methionine $[56,66]$, proline [67], isoleucine, valine [59], arginine [68], creatine [56,60] and histamine [69], are indirectly involved in a seizure.

In general, the mechanisms of all mentioned compounds are related to brain activities. Therefore, the plasma concentrations should be interpreted with care. The relation brain-plasma concentration depends on the blood-brain barrier permeability of the compounds and may additionally be influenced by specific transporters. For instance, a study reported comparable glutamine concentrations in the hippocampus of kindled and non-kindled rats [57], while lower glutamine concentrations were reported in serum of patients with seizures [5]. In our study, glutamine was identified as a marker for the second evoked seizure. However, the number of similar metabolic changes in the hippocampus and plasma is limited [8]. This is due to the fact that epilepsy and seizures are brain related disorders, but there is an increased need for peripheral markers as they allow a fast screening. The timing of the experimental design, type of research samples may influence the outcomes and even differences in analytical platforms can affect the findings, but in general most of the identified metabolites show similarities with other observations in literature.

\section{Conclusion and future perspectives}

In this study, we have demonstrated the utility of our approach, which includes sampling, sample pretreatment and CE-MS analysis of low-volume plasma samples, for finding metabolic consequences of evoked seizures. Seizures were induced in mice by $6 \mathrm{~Hz}$ corneal stimulation and plasma samples were collected by capillary microsampling before and after stimulation. The $6 \mathrm{~Hz}$ electrical stimulation was repeated after a 13 days wash-out period to investigate whether the same metabolic changes are observed in naive and previously stimulated mice.

After both evoked seizures a decrease in plasma concentrations of methionine, lysine, glycine, phenylalanine, 3-methyladenine, citrulline and histidine is observed. The decreases of glycine, citrulline and histidine are also observed in previously reported studies. However, in general most amino acids showed a less pronounced decrease in plasma after a second seizure compared to the first.

The reported decreased levels of isoleucine, serine, histidine, citrulline, proline and tryptophan after a seizure are similar to observations in literature of a chemo convulsive animal model for seizures. Therefore, these markers are related to some seizure activities and are independent of the obtained seizure model. Additionally, the decreased concentrations of alanine, arginine, valine and asparagine were also previously observed in human hippocampal tissue studies or serum samples. No significant differences between the basal plasma levels and those obtained after a 13 days wash-out period were observed, suggesting the effectiveness of the wash-out period.

Besides the similar observations of the previously described changes in literature, some contradictions are present. As an example, the observed changes of the monoamines and their precursors. Therefore, it would be useful to further investigate and validate their role in different animal models (electrical and chemo convulsive) as in humans by different analytical techniques. Additionally, some compounds were not yet identified. Knowledge on their identity could define or clarify their role in seizures.

In general, most metabolic markers that were identified by our study are amino acids. This could be attributed to the employed CE-MS separation conditions, which are specifically suited for the profiling of basic metabolites, including compound classes, such as amino acids, amines and nucleosides. A next step would be to use also CE-MS for the profiling of acidic metabolites, i.e. organic acids, nucleotides and sugar phosphates, in the same samples and to assess whether metabolic changes can be detected which might be linked to seizures. For lipidlike compounds, we would like to investigate the potential of nonaqueous CE-MS in the future.

Overall, our study emphasized the suitability of CE-MS to analyze volume-restricted plasma samples with an acceptable variation. 
Additionally, the method is sensitive enough to measure a suitable profile of polar components, differentiating the samples before and after an evoked seizure and define some amino acids involved in evoked seizures.

\section{Declaration of interests}

The authors declare that they have no known competing financial interests or personal relationships that could have appeared to influence the work reported in this paper.

\section{CRediT authorship contribution statement}

Karen Segers: Investigation, Formal analysis, Writing - original draft. Wei Zhang: Investigation, Formal analysis, Writing - original draft. Najat Aourz: Formal analysis, Writing - review \& editing. Jana Bongaerts: Formal analysis, Writing - review \& editing. Sven Declerck: Formal analysis, Writing - review \& editing. Debby Mangelings: Resources, Writing - review \& editing. Thomas Hankemeier: Resources, Writing - review \& editing. Dimitri De Bundel: Methodology, Writing - review \& editing. Yvan Vander Heyden: Methodology, Resources, Writing - review \& editing. Ilse Smolders: Conceptualization, Methodology, Resources, Writing - review \& editing. Rawi Ramautar: Conceptualization, Methodology, Resources, Writing - original draft, Supervision. Ann Van Eeckhaut: Conceptualization, Methodology, Resources, Writing - original draft, Supervision.

\section{Acknowledgments}

This work was supported by a Travel Grant of the Research Foundation Flanders (FWO) under Grant number V433318 N for K. Segers. W. Zhang acknowledges the China Scholarship Council (CSC, No. 201507060011) for supporting his PhD project at Leiden University. R. Ramautar acknowledges the Netherlands Organization for Scientific Research (NWO) for support of the research with a Vidi grant. The authors would also thank N. Drouin for the support with the ROMANCE software as well as L. Walrave, A. Buckinx, Y. Van Den Herrewegen, A. Pierre and W. Allaoui for their excellent help with the animal experiments. Furthermore, we thank L. Dillen, T. Gevers, P. Anthonissen, H. Sieltjens, A. Vromans and C. Sas from Janssen Pharmaceutica - Johnson \& Johnson to guide us with the use of capillary microsampling.

\section{References}

[1] C.A. Chamberlain, V.Y. Rubio, T.J. Garrett, Impact of matrix effects and ionization efficiency in non-quantitative untargeted metabolomics, Metabolomics 15 (10) (2019) 135, https://doi.org/10.1007/s11306-019-1597-z.

[2] R. Shyti, I. Kohler, B. Schoenmaker, R.J.E. Derks, M.D. Ferrari, E.A. Tolner, O.A. Mayboroda, A.M.J.M. van den Maagdenberg, Plasma metabolic profiling after cortical spreading depression in a transgenic mouse model of hemiplegic migraine by capillary electrophoresis - mass spectrometry, Mol. Biosyst. 11 (5) (2015) 1462-1471, https://doi.org/10.1039/c5mb00049a.

[3] R.S. Fisher, C. Acevedo, A. Arzimanoglou, A. Bogacz, J.H. Cross, C.E. Elger, J. Engel Jr., L. Forsgren, J.A. French, M. Glynn, ILAE official report: a practical clinical definition of epilepsy, Epilepsia 55 (4) (2014) 475-482, https://doi.org/10.1111/ epi.12550.

[4] P. Bazzigaluppi, A. Ebrahim Amini, I. Weisspapir, B. Stefanovic, L.P. Carlen, Hungry neurons: metabolic insights on seizure dynamics, Int. J. Mol. Sci. 18 (11) (2017) 2269, https://doi.org/10.3390/ijms18112269.

[5] D. Wang, X. Wang, J. Kong, J. Wu, M. Lai, GC-MS-Based metabolomics discovers a shared serum metabolic characteristic among three types of epileptic seizures, Epilepsy Res. 126 (2016) 83-89, https://doi.org/10.1016/j.eplepsyres.2016.07. 003.

[6] D.I. Walker, K. Perry-Walker, R.H. Finnell, K.D. Pennell, V. Tran, R.C. May, T.F. McElrath, K.J. Meador, P.B. Pennell, D.P. Jones, Metabolome-wide association study of anti-epileptic drug treatment during pregnancy, Toxicol. Appl. Pharmacol. 363 (2019) 122-130, https://doi.org/10.1016/j.taap.2018.12.001.

[7] S. Li, D. Song, W. Huang, Z. Li, Z. Liu, In situ imaging of cysteine in the brains of mice with epilepsy by a near-infrared emissive fluorescent probe, Anal. Chem. 92
(3) (2020) 2802-2808, https://doi.org/10.1021/acs.analchem.9b05211.

[8] S. Heischmann, K. Quinn, C. Cruickshank-Quinn, L.P. Liang, R. Reisdorph, N. Reisdorph, M. Patel, Exploratory metabolomics profiling in the kainic acid rat model reveals depletion of 25-hydroxyvitamin D3 during epileptogenesis, Sci. Rep. 6 (2016) 1-14, https://doi.org/10.1038/srep31424.

[9] X. Tan, Z. Tu, W. Han, X. Song, L. Cheng, H. Chen, S. Tu, P. Li, W. Liu, L. Jiang, Anticonvulsant and neuroprotective effects of dexmedetomidine on pilocarpineinduced status epilepticus in rats using a metabolomics approach, Med. Sci. Mon. Int. Med. J. Exp. Clin. Res. 25 (2019) 2066-2078, https://doi.org/10.12659/MSM. 912283.

[10] Y. Kanawaku, K. Hirakawa, K. Koike, J. Kanetake, Y. Ohno, Pattern recognition analysis of proton nuclear magnetic resonance spectra of postmortem cerebrospina fluid from rats with drug-induced seizure or coma, Leg. Med. 25 (2017) 52-58, https://doi.org/10.1016/j.legalmed.2017.01.004.

[11] P. Li, D.D. Wei, J.S. Wang, M.H. Yang, L.Y. Kong, (1)H NMR metabolomics to study the effects of diazepam on anisatin induced convulsive seizures, J. Pharmaceut. Biomed. Anal. 117 (2016) 184-194, https://doi.org/10.1016/j.jpba.2015.08.029.

[12] S. Carmody, L. Brennan, Effects of pentylenetetrazole-induced seizures on metabolomic profiles of rat brain, Neurochem. Int. 56 (2010) 340-344, https://doi.org/ 10.1016/j.neuint.2009.11.004.

[13] M. Urban, D.P. Enot, G. Dallmann, L. Körner, V. Forcher, P. Enoh, T. Koal, M. Keller, H.-P. Deigner, Complexity and pitfalls of mass spectrometry-based targeted metabolomics in brain research, Anal. Biochem. 406 (2) (2010) 124-131, https://doi. org/10.1016/j.ab.2010.07.002.

[14] M.C. Hogg, R. Raoof, H. El Naggar, N. Monsefi, N. Delanty, D.F. O'Brien, S. Bauer, F. Rosenow, D.C. Henshall, J.H.M. Prehn, Elevation of plasma tRNA fragments precedes seizures in human epilepsy, J. Clin. Invest. 129 (7) (2019) 2946-2951, https://doi.org/10.1172/JCI126346.

[15] H. McArdle, E.M. Jimenez-Mateos, R. Raoof, E. Carthy, D. Boyle, H. ElNaggar, N. Delanty, H. Hamer, M. Dogan, T. Huchtemann, "TORNADO"-Theranostic OneStep RNA Detector; microfluidic disc for the direct detection of microRNA-134 in plasma and cerebrospinal fluid, Sci. Rep. 7 (1) (2017) 1-11, https://doi.org/10. 1038/s41598-017-01947-2.

[16] J. Detour, C. Bund, C. Behr, H. Cebula, E.A. Cicek, M.P. Valenti-Hirsch, B. Lannes, B. Lhermitte, A. Nehlig, P. Kehrli, F. Proust, E. Hirsch, I.J. Namer, Metabolomic characterization of human hippocampus from drug-resistant epilepsy with mesial temporal seizure, Epilepsia 59 (2018) 607-616, https://doi.org/10.1111/epi. 14000.

[17] F. Murgia, A. Muroni, M. Puligheddu, L. Polizzi, L. Barberini, G. Orofino, P. Solla, S. Poddighe, F. Del Carratore, J.L. Griffin, L. Atzori, F. Marrosu, Metabolomics as a tool for the characterization of drug-resistant epilepsy, Front. Neurol. 8 (2017) 459, https://doi.org/10.3389/fneur.2017.00459.

[18] M. Al Zweiri, G.J. Sills, J.P. Leach, M.J. Brodie, C. Robertson, D.G. Watson, J.A. Parkinson, Response to drug treatment in newly diagnosed epilepsy: a pilot study of (1)H NMR- and MS-based metabonomic analysis, Epilepsy Res. 88 (2010) 189-195, https://doi.org/10.1016/j.eplepsyres.2009.11.005.

[19] F. Raynaud, J. Roberts, Capillary microsampling of mouse blood in pre-clinical studies: an alternative to dried blood spot sampling, J. Bioanal. Biomed. 8 (2) (2019) 28-33, https://doi.org/10.4172/1948-593X.1000149.

[20] A.A. Raje, V. Mahajan, V.V. Pathade, K. Joshi, A. Gavali, A. Gaur, V. Kandikere, Capillary microsampling in mice: effective way to move from sparse sampling to serial sampling in pharmacokinetics profiling, Xenobiotica (2019) 1-7, https://doi. org/10.1080/00498254.2019.1683259.

[21] S.R. Patel, P. Bryan, N. Spooner, P. Timmerman, E. Wickremsinhe, Microsampling for quantitative bioanalysis, an industry update: output from an AAPS/EBF survey, Bioanalysis 11 (7) (2019) 619-628, https://doi.org/10.4155/bio-2019-0019.

[22] I. Xie, Y. Xu, M. Anderson, M. Wang, L. Xue, S. Breidinger, D. Goykhman, E.J. Woolf, K.P. Bateman, Extractability-mediated stability bias and hematocrit impact: high extraction recovery is critical to feasibility of volumetric adsorptive microsampling (VAMS) in regulated bioanalysis, J. Pharmaceut. Biomed. Anal. 156 (2018) 58-66, https://doi.org/10.1016/j.jpba.2018.04.001.

[23] J.D. Freeman, L.M. Rosman, J.D. Ratcliff, P.T. Strickland, D.R. Graham, E.K. Silbergeld, State of the science in dried blood spots, Clin. Chem. 64 (4) (2018) 656-679, https://doi.org/10.1373/clinchem.2017.275966.

[24] T. Verhaeghe, L. Dillen, H. Stieltjes, L. Zwart, B. Feyen, L. Diels, A. Vroman, P. Timmerman, The application of capillary microsampling in GLP toxicology studies, Bioanalysis 9 (7) (2017) 531-540, https://doi.org/10.4155/bio-2016-0297.

[25] T. Verhaeghe, L. Dillen, H. Stieltjes, L.d. Zwart, B. Feyen, Comparison of toxicokinetic parameters of a drug and two metabolites following traditional and capillary microsampling in rat, Bioanalysis 11 (13) (2019) 1233-1242, https://doi. org/10.4155/bio-2019-0085.

[26] Z. Cobb, M. Rohde, I. Love, V. Boutet, K. Schroeter, G. Hawthorne, L. Dillen, M. Barfield, A. Kandira, M. Kranenborgh, Feedback from the European Bioanalysis Forum liquid microsampling consortium: capillary liquid microsampling and assessment of homogeneity of the resultant samples, Bioanalysis 11 (6) (2019) 525-532, https://doi.org/10.4155/bio-2019-0017.

[27] W. Zhang, F. Guled, T. Hankemeier, R. Ramautar, Utility of sheathless capillary electrophoresis-mass spectrometry for metabolic profiling of limited sample amounts, J. Chromatogr. B Analyt. Technol. Biomed. Life Sci. 1105 (2018) 10-14, https://doi.org/10.1016/j.jchromb.2018.

[28] R. Ramautar, Sheathless capillary electrophoresis-mass spectrometry for the profiling of charged metabolites in biological samples, Methods Mol. Biol. 1738 (2018) 183-192, https://doi.org/10.1007/978-1-4939-7643-0 12.

[29] P. Miggiels, B. Wouters, G.J.P. van Westen, A.-C. Dubbelman, T. Hankemeier, Novel technologies for metabolomics: more for less, Trends Anal. Chem. 120 (2018) 1253223, https://doi.org/10.1016/j.trac.2018.11.021. 
[30] E. Sanchez-Lopez, G.S.M. Kammeijer, A.L. Crego, M.L. Marina, R. Ramautar, D.J.M. Peters, O.A. Mayboroda, Sheathless CE-MS based metabolic profiling of kidney tissue section samples from a mouse model of Polycystic Kidney Disease, Sci. Rep. 9 (2019) 1-9, https://doi.org/10.1038/s41598-018-37512-8.

[31] A. Hirayama, H. Abe, N. Yamaguchi, S. Tabata, M. Tomita, T. Soga, Development of a sheathless CE-ESI-MS interface, Electrophoresis 39 (11) (2018) 1382-1389, https://doi.org/10.1002/elps.201800017.

[32] P. Fang, J.Z. Pan, Q. Fang, A robust and extendable sheath flow interface with minimal dead volume for coupling CE with ESI-MS, Talanta 180 (2018) 376-382, https://doi.org/10.1016/j.talanta.2017.12.046.

[33] S. Harada, A. Hirayama, Q. Chan, A. Kurihara, K. Fukai, M. Iida, S. Kato, D. Sugiyama, K. Kuwabara, A. Takeuchi, M. Akiyama, T. Okamura, T.M.D. Ebbels, P. Elliott, M. Tomita, A. Sato, C. Suzuki, M. Sugimoto, T. Soga, T. Takebayashi, Reliability of plasma polar metabolite concentrations in a large-scale cohort study using capillary electrophoresis-mass spectrometry, PloS One 13 (1) (2018) e0191230, , https://doi.org/10.1371/journal.pone.0191230.

[34] W. Zhang, K. Segers, D. Mangelings, A. Van Eeckhaut, T. Hankemeier, Y. Vander Heyden, R. Ramautar, Assessing the suitability of capillary electrophoresis-mass spectrometry for biomarker discovery in plasma-based metabolomics, Electrophoresis 40 (2019) 2309-2320, https://doi.org/10.1002/elps.201900126.

[35] N. Drouin, J. Pezzatti, Y. Gagnebin, V. González-Ruiz, J. Schappler, S. Rudaz, Effective mobility as a robust criterion for compound annotation and identification in metabolomics: toward a mobility-based library, Anal. Chim. Acta 1032 (2018) 178-187, https://doi.org/10.1016/j.aca.2018.05.063.

[36] W.C. Brown, D.O. Schiffman, E.A. Swinyard, L.S. Goodman, Comparative assay of an antiepileptic drugs by psychomotor seizure test and minimal electroshock threshold test, J. Pharmacol. Exp. Therapeut. 107 (1953) 273-283.

[37] M.E. Barton, B.D. Klein, H.H. Wolf, H.S. White, Pharmacological characterization of the $6 \mathrm{~Hz}$ psychomotor seizure model of partial epilepsy, Epilepsy Res. 47 (2001) 217-227, https://doi.org/10.1016/s0920-1211(01)00302-3.

[38] L. Walrave, K. Maes, J. Coppens, E. Bentea, A. Van Eeckhaut, A. Massie, J. Van Liefferinge, I. Smolders, Validation of the $6 \mathrm{~Hz}$ refractory seizure mouse model for intracerebroventricularly administered compounds, Epilepsy Res. 115 (2015) 67-72, https://doi.org/10.1016/j.eplepsyres.2015.06.003.

[39] G. Albertini, L. Walrave, T. Demuyser, A. Massie, D. De Bundel, I. Smolders, $6 \mathrm{~Hz}$ corneal kindling in mice triggers neurobehavioral comorbidities accompanied by relevant changes in c-Fos immunoreactivity throughout the brain, Epilepsia 59 (1) (2018) 67-78, https://doi.org/10.1111/epi.13943.

[40] E. Takach, T. O'Shea, H. Liu, High-throughput quantitation of amino acids in rat and mouse biological matrices using stable isotope labeling and UPLC-MS/MS analysis, J. Chromatogr. B Analyt. Technol. Biomad. Life Sci. 964 (2014) 180-190, https:// doi.org/10.1016/j.jchromb.2014.04.043.

[41] J.C. Lindon, J.K. Nicholson, E. Holmes, H.C. Keun, A. Craig, J.T.M. Pearce, S.J. Bruce, N. Hardy, S.-A. Sansone, H. Antti, P. Jonsson, C. Daykin, M. Navarange, R.D. Beger, E.R. Verheij, A. Amberg, D. Baunsgaard, G.H. Cantor, L. LehmanMcKeeman, M. Earll, S. Wold, E. Johansson, J.N. Haselden, K. Kramer, C. Thomas, J. Lindberg, I. Schuppe-Koistinen, I.D. Wilson, M.D. Reily, D.G. Robertson, H. Senn, A. Krotzky, S. Kochhar, J. Powell, F. van der Ouderaa, R. Plumb, H. Schaefer, M. Spraul, Summary recommendations for standardization and reporting of metabolic analyses, Nat. Biotechnol. 23 (7) (2005) 833-838, https://doi.org/10.1038/ nbt0705-833.

[42] R. Madsen, T. Lundstedt, J. Trygg, Chemometrics in metabolomics-a review in human disease diagnosis, Anal. Chim. Acta 659 (1-2) (2010) 23-33, https://doi. org/10.1016/j.aca.2009.11.042.

[43] E. Ortiz-Villanueva, F. Benavente, B. Pina, V. Sanz-Nebot, R. Tauler, J. Jaumot, Knowledge integration strategies for untargeted metabolomics based on MCR-ALS analysis of CE-MS and LC-MS data, Anal. Chim. Acta 978 (2017) 10-23, https://doi. org/10.1016/j.aca.2017.04.049.

[44] E. Gorrochategui, J. Jaumot, R. Tauler, A protocol for LC-MS metabolomic data processing using chemometric tools, protocol exhange, https://doi.org/10.13140/ RG.2.1.2285.7049, (2015).

[45] J. Jaumot, A. de Juan, R. Tauler, MCR-ALS GUI 2.0: new features and applications, Chemometr. Intell. Lab. Syst. 140 (2015) 1-12, https://doi.org/10.1016/j. chemolab.2014.10.003.

[46] T. Zhang, D.G. Watson, Evaluation of the technical variations and the suitability of a hydrophilic interaction liquid chromatography-high resolution mass spectrometry (ZIC-pHILIC-Exactive orbitrap) for clinical urinary metabolomics study, J. Chromatogr. B Analyt. Technol. Biomed. Life Sci. 1022 (2016) 199-205, https:// doi.org/10.1016/j.jchromb.2016.04.017.

[47] V. González-Ruiz, Y. Gagnebin, N. Drouin, S. Codesido, S. Rudaz, J. Schappler, ROMANCE: a new software tool to improve data robustness and feature identification in CE-MS metabolomics, Electrophoresis 39 (9-10) (2018) 1222-1232, https://doi.org/10.1002/elps.201700427.

[48] C.A. Smith, G. O'Maille, E.J. Want, C. Qin, S.A. Trauger, T.R. Brandon,
D.E. Custodio, R. Abagyan, G. Siuzdak, METLIN: a metabolite mass spectral database, Ther. Drug Monit. 27 (2005) 747-751, https://doi.org/10.1097/01.ftd. 0000179845.53213 .39$.

[49] I. Laponogov, N. Sadawi, D. Galea, R. Mirnezami, K.A. Veselkov, ChemDistiller: an engine for metabolite annotation in mass spectrometry, Bioinformatics 34 (2018) 2096-2102, https://doi.org/10.1093/bioinformatics/bty080.

[50] E. Gorrochategui, J. Jaumot, S. Lacorte, R. Tauler, Data analysis strategies for targeted and untargeted LC-MS metabolomic studies: overview and workflow, Trends Anal. Chem. 82 (2016) 425-442, https://doi.org/10.1016/j.trac.2016.07. 004.

[51] F. Zheng, Z.A. Xia, Y.F. Zeng, J.K. Luo, P. Sun, H.J. Cui, Y. Wang, T. Tang, Y.T. Zhou, Plasma metabolomics profiles in rats with acute traumatic brain injury, PloS One 12 (8) (2017) e0182025, , https://doi.org/10.1371/journal.pone. 0182025.

[52] R. Gosselin, D. Rodrigue, C. Duchesne, A Bootstrap-VIP approach for selecting wavelength intervals in spectral imaging applications, Chemometr. Intell. Lab. Syst. 100 (2010) 12-21, https://doi.org/10.1016/j.chemolab.2009.09.005.

[53] M.J. During, D.D. Spencer, Extracellular hippocampal glutamate and spontaneous seizure in the conscious human brain, Lancet 341 (8861) (1993) 1607-1610, https://doi.org/10.1016/0140-6736(93)90754-5.

[54] K.G. Haglid, S. Wang, Y. Qiner, A. Hamberger, Excitotoxicity. Experimental correlates to human epilepsy, Mol. Neurobiol. 9 (1994) 259-263.

[55] C.L. Wilson, N.T. Maidment, M.H. Shomer, E.J. Behnke, L. Ackerson, I. Fried, J. Engel Jr., Comparison of seizure related amino acid release in human epileptic hippocampus versus a chronic, kainate rat model of hippocampal epilepsy, Epilepsy Res. 26 (1) (1996) 245-254, https://doi.org/10.1016/s0920-1211(96)00057-5.

[56] L. Boguszewicz, E. Jamroz, M. Ciszek, E. Emich-Widera, M. Kijonka, T. Banasik, A. Skorupa, M. Sokol, NMR-based metabolomics in pediatric drug resistant epilepsy - preliminary results, Sci. Rep. 9 (2019) 1-15, https://doi.org/10.1038/s41598019-51337-z.

[57] J. Szyndler, P. Maciejak, D. Turzynska, A. Sobolewska, M. Lehner, E. Taracha, J. Walkowiak, A. Skorzewska, A. Wislowska-Stanek, A. Hamed, A. Bidzinski, A. Plaznik, Changes in the concentration of amino acids in the hippocampus of pentylenetetrazole-kindled rats, Neurosci. Lett. 439 (3) (2008) 245-249, https:// doi.org/10.1016/j.neulet.2008.05.002.

[58] T. Eid, M.J. Thomas, D.D. Spencer, E. Rundén-Pran, J.C.K. Lai, G.V. Malthankar, J.H. Kim, N.C. Danbolt, O.P. Ottersen, N.C. de Lanerolle, Loss of glutamine synthetase in the human epileptogenic hippocampus: possible mechanism for raised extracellular glutamate in mesial temporal lobe epilepsy, Lancet 363 (9402) (2004) 28-37, https://doi.org/10.1016/s0140-6736(03)15166-5.

[59] E. Bejarano, J.A. Rodriguez-Navarro, Autophagy and amino acid metabolism in the brain: implications for epilepsy, Amino Acids 47 (10) (2015) 2113-2126, https:// doi.org/10.1007/s00726-014-1822-z.

[60] G. Wu, Amino acids: metabolism, functions, and nutrition, Amino Acids 37 (1) (2009) 1-17, https://doi.org/10.1007/s00726-009-0269-0.

[61] P.C. Jobe, J.W. Dailey, Aspartame and seizures, Amino Acids 4 (1993) 197-235, https://doi.org/10.1007/BF00805824.

[62] G.R. Wenger, R.E. Stitzel, C.R. Craig, The role of biogenic amines in the reserpineinduced alteration of minimal electroshock seizure thresholds in the mouse, Neuropharmacology 12 (7) (1973) 693-703, https://doi.org/10.1016/0028 3908(73)90122-6.

[63] D. Svob Strac, N. Pivac, I.J. Smolders, W.A. Fogel, P. De Deurwaerdere, G. Di Giovanni, Monoaminergic mechanisms in epilepsy may offer innovative therapeutic opportunity for monoaminergic multi-target drugs, Front. Neurosci. 10 (2016) 492, https://doi.org/10.3389/fnins.2016.00492.

[64] M.A. Kurian, P. Gissen, M. Smith, S. Heales Jr., P.T. Clayton, The monoamine neurotransmitter disorders: an expanding range of neurological syndromes, Lancet Neurol. 10 (8) (2011) 721-733, https://doi.org/10.1016/S1474-4422(11)70141-7.

[65] S.M. Lasley, Roles of neurotransmitter amino acids in seizure severity and experience in the genetically epilepsy-prone rat, Brain Res. 560 (1-2) (1991) 63-70, https://doi.org/10.1016/0006-8993(91)91215-m.

[66] J. Yin, W. Ren, G. Yang, J. Duan, X. Huang, R. Fang, C. Li, T. Li, Y. Yin, Y. Hou, S.W. Kim, G. Wu, L-Cysteine metabolism and its nutritional implications, Mol. Nutr. Food Res. 60 (1) (2016) 134-146, https://doi.org/10.1002/mnfr.201500031.

[67] Z.E. Pontes, L.S. Oliveira, C.S. Baveresco, E.L. Streck, C.S. Dutra-Filho, M. Wajner, C.M. Wannmacher, A.T. Wyse, Proline administration decreases $\mathrm{Na}^{+}, \mathrm{K}^{+}$-ATPase activity in the synaptic plasma membrane from cerebral cortex of rats, Metab. Brain Dis. 14 (4) (1999) 265-272, https://doi.org/10.1023/a:1020789109913.

[68] K. Hirata, Y. Akita, N. Povalko, J. Nishioka, S. Yatsuga, T. Matsuishi, Y. Koga, Effect of l-arginine on synaptosomal mitochondrial function, Brain Dev. 30 (4) (2008) 238-245, https://doi.org/10.1016/j.braindev.2007.08.007.

[69] H. Kakinoki, K. Ishizawa, M. Fukunaga, Y. Fujii, C. Kamei, The effects of histamine H3-receptor antagonists on amygdaloid kindled seizures in rats, Brain Res. Bull. 46 (5) (1998) 461-465, https://doi.org/10.1016/s0361-9230(98)00048-3. 